Mon. Not. R. Astron. Soc. 000, 1-?? (2008) Printed 1 November $2018 \quad$ (MN LATEX style file v2.2)

\title{
Cosmological Constraints from calibrated Yonetoku and Amati relation suggest Fundamental plane of Gamma-ray bursts
}

\author{
Ryo Tsutsui ${ }^{1 \star}$, Takashi Nakamura ${ }^{1}$, Daisuke Yonetoku²,Toshio Murakami², \\ Yoshiki Kodama ${ }^{2}$, and Keitaro Takahashi ${ }^{3}$ \\ ${ }^{1}$ Department of Physics, Kyoto University, Kyoto 606-8502, Japan \\ ${ }^{2}$ Department of Physics, Faculty of Science, Kanazawa University, Kakuma, Kanazawa, Ishikawa 920-1192, Japan \\ ${ }^{3}$ Yukawa Institute for Theoretical Physics, Kyoto University, Kyoto 606-8502, Japan
}

1 November 2018

\begin{abstract}
We consider two empirical relations using data only from the prompt emission of Gamma-Ray Bursts (GRBs), the peak energy $\left(E_{p}\right)$ - peak luminosity $\left(L_{p}\right)$ relation (so called Yonetoku relation) and the $E_{p}$-isotropic energy $\left(E_{\text {iso }}\right)$ relation (so called Amati relation). Both relations show high correlation degree, but they also have larger dispersion around the best fit function rather than the statistical uncertainty. Then we first investigated the correlation between the residuals of $L_{p}$ and $E_{i s o}$ from the best function, and found that a partial linear correlation degree is quite small of $\rho_{L_{p} E_{i s s} \cdot E_{p}}=0.379$. This fact indicates that some kinds of independence may exist between Amati and Yonetoku relation even if they are characterized by the same physical quantity $E_{p}$, and similar quantities $L_{p}$ and $E_{i s o}$ which mean the brightness of the prompt emission. Therefore we may have to recognize two relations as the independent distance indicators. From this point of view, we compare constraints on cosmological parameters, $\Omega_{m}$ and $\Omega_{\Lambda}$, using the Yonetoku and the Amati relation calibrated by low-redshift GRBs with $z<1.8$. We found that they are different in 1- $\sigma$ level, although they are still consistent in $2-\sigma$ level. In this paper, we introduce a luminosity time $T_{L}$ defined by $T_{L} \equiv E_{\text {iso }} / L_{p}$ as a hidden parameter to correct the large dispersion of the Yonetoku relation. A new relation is described as $\left(L_{p} / 10^{52} \mathrm{erg} \mathrm{s}^{-1}\right)=10^{-3.87 \pm 0.19}\left(E_{p} / \mathrm{keV}\right)^{1.82 \pm 0.08}\left(T_{L} / \mathrm{s}\right)^{-0.34 \pm 0.09}$. We succeeded in reducing the systematic error about $40 \%$ level, and might be regarded as "Fundamental plane" of GRBs. We show a possible radiation model for this new relation. Finally, applying the new relation to high-redshift GRBs with $1.8<z<5.6$, we obtain $\left(\Omega_{m}, \Omega_{\Lambda}\right)=\left(0.17_{-0.08}^{+0.15}, 1.21_{-0.61}^{+0.07}\right)$, which is consistent with the concordance cosmological model.
\end{abstract}

Key words:

gamma rays: bursts - gamma rays: observation

\section{INTRODUCTION}

In our previous papers Kodama et al. 2008; Tsutsui et al. 2009), we calibrated the relation between peak energy $E_{p}$ and peak luminosity $L_{p}$ of prompt GRB emission (so called Yonetoku relation (Yonetoku et al. 2004)) by 33 low-redshift GRBs $(z<1.62)$ whose luminosity distances were estimated from SNeIa (Riess et al. 2007; Wood-Vasey et al. 2007; Davis et al. 2007). Then we used the calibrated Yonetoku relation as a distance indicator like the period-

\footnotetext{
* E-mail: tsutsui@tap.scphys.kyoto-u.ac.jp (RT)
}

luminosity relation of Cepheid variables, and extended the Hubble diagram up to $z=5.6$. Tsutsui et al. (2009) showed that GRBs constrain cosmological parameters in a different way from SNeIa, and GRBs could be useful to probe cosmological expansion of high-redshift universe where no SNIa has been observed.

The Amati relation (Amati et al. 2002) is another relation for prompt emission property. It involves peak energy $E_{p}$ and isotropic energy $E_{\text {iso }}$, and was originally derived under a given set of cosmological parameters. Therefore, the circularity problem arises if one applies naively the Amati relation to determine cosmological parameters. To overcome 
this difficulty, in this paper, we first calibrate the Amati relation as we did for Yonetoku relation without assuming any cosmological models but using luminosity distance given by SNeIa for $z<1.8$.

Although the strong correlation between $E_{\text {iso }}$ and $L_{p}$ are generally confirmed, we found little trend between their data residuals of $\Delta L_{p}$ and $\Delta E_{\text {iso }}$ from the best fit function of each relation. We suggest statistical independence of Amati and Yonetoku relations while these two relations have the same parameter $E_{p}(\S-2)$.

Next, we will extend the Hubble diagram with the obtained relations and make constraints on density parameters, $\left(\Omega_{m}, \Omega_{\Lambda}\right)$. It is shown that the two Hubble diagrams differ systematically at high redshifts and, as a result, two different constraints are obtained $(\S-3)$. Although the difference is not so significant (1- $\sigma$ level), taking the relatively large systematic errors in the two relations themselves into consideration, it is suggested that there may be a hidden parameter which characterizes the prompt emission and reduces the systematic error of the distance indicator. We introduce the luminosity time $\left(T_{L} \equiv E_{\text {iso }} / L_{p}\right)$ as a possible hidden parameter to derive the $E_{p}-T_{L}-L_{p}$ relation, and put constraints on cosmological parameters $(\S-4)$. Finally we give some comments on other relations used as distance indicators in the past, and argue the advantage of our new relation (§-5). Throughout the paper, we fix the current Hubble parameter as $H_{0}=66 \mathrm{~km} \mathrm{~s}^{-1} \mathrm{Mpc}^{-1}$.

We note that, in whole this paper, tentatively we do not take possible selection effects and evolution effects on relations into accounts although a hot debate (Butler et al. 2007; Li 2007; Basilakos \& Perivolaropoulos 2008) exists. We think that the final resolution needs the increase of the events with enough well determined parameters such as redshifts, $E_{p}, L_{p}$ and $E_{\text {iso }}$ since we should divide all data into several sub groups to check possible selection effects and evolution effects.

\section{CALIBRATION OF AMATI RELATION AT LOW REDSHIFTS}

The typical spectrum of the prompt emission of GRBs can be expressed as exponentially connected broken power-law, so called Band function (Band et al. 1993). Then we can determine spectral peak energy $E_{p}$, corresponding to the photon energy at maximum in $\nu F_{\nu}$ spectra. There are two empirical relations that relate prompt emission property with $E_{p} . E_{p}-E_{\text {iso }}$ relation is the first one found by Amati et al. (2002), which connects $E_{p}$ with the isotropic equivalent energy $E_{\text {iso. }}$. The second one is $E_{p}-L_{p}$ relation found by Yonetoku et al. (2004) which was used in our previous papers (Kodama et al. 2008; Tsutsui et al. 2009).

We first calibrate Amati relation in the same way as in our previous papers (Kodama et al. 2008; Tsutsui et al. 2009) and analyze the correlation of the residuals of GRB data from the relations and the partial correlation coefficient. If there are a small degree of correlation between $L_{p}$ and $E_{\text {iso }}$ after removing the effect of $E_{p}$, it suggest the possible independence of the distance indicators, and might suggest the existence of a hidden parameter common to each relation.

We found an empirical formula for the luminosity dis- tance as a function of redshift from 192 SNeIa observations (Riess et al. 2007; Davis et al. 2007; Wood-Vasev et al. 2007),

$$
\frac{d_{L}}{10^{27} \mathrm{~cm}}=6.96 \times z^{1.79}+14.79 \times z^{1.02} .
$$

The reduced chi-square of the formula is $\chi_{\nu}^{2}=0.995$. Note here that the formula is not unique and a different formula is possible. Note also that we do not assume any cosmological models at this stage, but simply assume that the Type Ia supernovae are the standard candles for $0.168<z<1.755$. Furthermore, we neglect the errors in Eq. (11) in the following analysis, which leads to the underestimation of errors in cosmological parameters. Our purpose here is to compare distance indicators and find a better indicator so that this neglect would be reasonable.

We apply this formula to 31 low redshifts GRBs within $z<1.6$. ( For details of our data, see Kodama et al. (2008).) In Fig. 1 we show the peak energy $E_{p}$ and the isotropic energy $E_{\text {iso }}$ of 31 GRBs with $z<1.62$. The solid line is the calibrated Amati relation given by,

$$
\frac{E_{\text {iso }}}{10^{52} \mathrm{erg}}=10^{-3.87 \pm 0.33}\left(\frac{E_{p}}{1 \mathrm{keV}}\right)^{2.01 \pm 0.14},
$$

where the statistical errors are indicated and $E_{\text {iso }}=$ $4 \pi d_{L}^{2} S_{\mathrm{bol}} /(1+z)$ where $S_{\mathrm{bol}}$ is the bolometric fluence estimated in 1-10000 keV energy range in GRB rest frame. The Pearson correlation coefficient is 0.943 and the reduced chi-square is $\chi^{2}=28.5 / 29$ with the systematic error $\sigma_{\text {sys }}=0.35$. Here we include not only errors in $E_{\text {iso }}$ but also errors in $E_{p}$, and the systematic error of this relation $\sigma_{\text {sys }}$, so the chi-square function is defined as $\chi^{2}(A, B)=\Sigma\left(\log E_{\text {iso }}^{\text {obs }}-A-B \log E_{p}\right)^{2} /\left(\sigma_{\text {meas }}^{2}+\sigma_{\text {sys }}^{2}\right)$ where the weighting factor $\sigma_{\text {meas }}^{2}=\sigma_{\log E_{\text {iso }}}^{2}+B^{2} \sigma_{\log E_{p}}^{2}$. The value of $\sigma_{\text {sys }}$ can be estimated by the value such that a $\chi^{2}$ fit to the Amati relation calibration produces a reduced $\chi^{2}$ of unity.

This kind of systematic error was also found in the Yonetoku relation (Kodama et al. 2008),

$\frac{L_{p}}{10^{52} \mathrm{erg} \mathrm{s}^{-1}}=10^{-3.95 \pm 0.27}\left(\frac{E_{p}}{1 \mathrm{keV}}\right)^{1.73 \pm 0.11}$

where $L_{p}$ is 1 -second peak luminosity. This Yonetoku relation is slightly different from that in the previous work because we include not only $L_{p}$ error but also $E_{p}$ error, and the systematic error in this paper. The Pearson correlation coefficient is 0.948 , the reduced chi-square is $\chi^{2}=30.6 / 31$, and the systematic error is $\sigma_{\text {sys }}=0.27$. The Amati relation has slightly larger systematic error than the Yonetoku relation and, in both cases, systematic errors are significantly larger than measurement errors.

Fig. 2] shows the correlation between two residuals which are defined as $\Delta L_{p} \equiv\left(\log L_{p}^{\text {obs }}-\log L_{p}^{\text {exp }}\right)$ and $\Delta E_{\text {iso }} \equiv$ $\left(\log E_{\text {iso }}^{\text {obs }}-\log E_{\text {iso }}^{\text {exp }}\right)$. Here, $L_{p}^{\text {obs }}\left(E_{\text {iso }}^{\text {obs }}\right)$ is the observed value, and $L_{p}^{\exp }\left(E_{\mathrm{iso}}^{\exp }\right)$ is the expected quantity from Yonetoku and Amati relations for observed $E_{p}$, respectively. We can see that there is no correlation between $\Delta L_{p}$ and $\Delta E_{i s o}$. We performed a linear partial correlation test for these 31 samples, and found the partial correlation coefficient is quite small of $\rho_{L_{p}} E_{i s o} \cdot E_{p}=0.379$. Here $\rho_{12.3}$ means the correlation coefficient between the first and the second parameters after fixing the third parameter which is controlling two cor- 


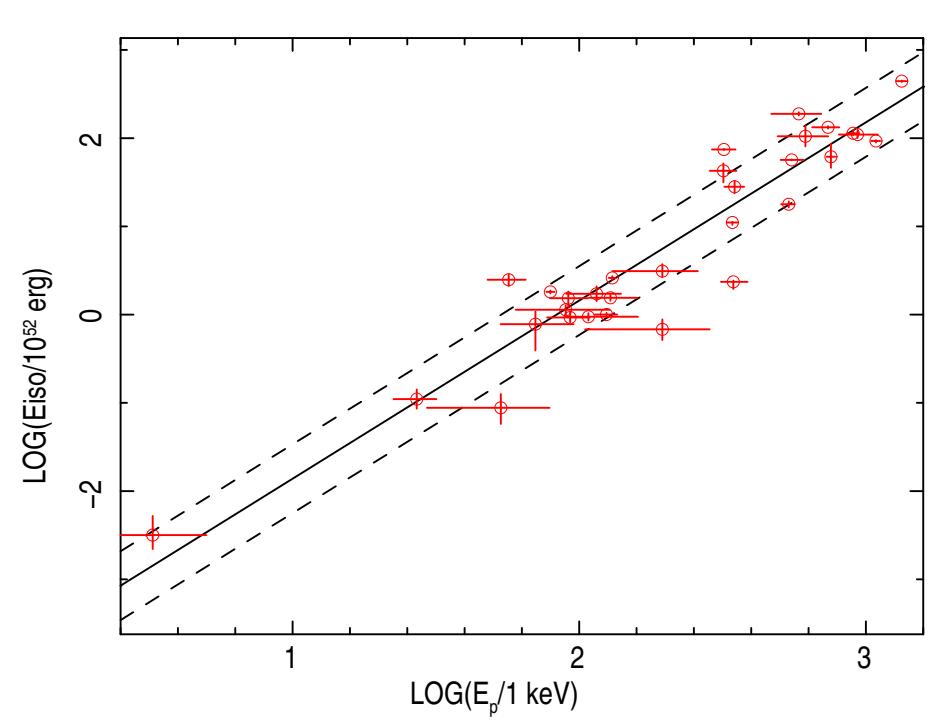

Figure 1. The peak energy $\left(E_{p}\right)$ and isotropic energy $\left(E_{\text {iso }}\right)$ of 31 GRBs with $z<1.62$. The solid line is the calibrated Amati relation given by $\mathrm{Eq}(2)$ and dashed lines represent the 1-sigma region. The systematic error of this relation is reduced to $\sigma_{s y s}=$ 0.35 which is larger than measurement errors.

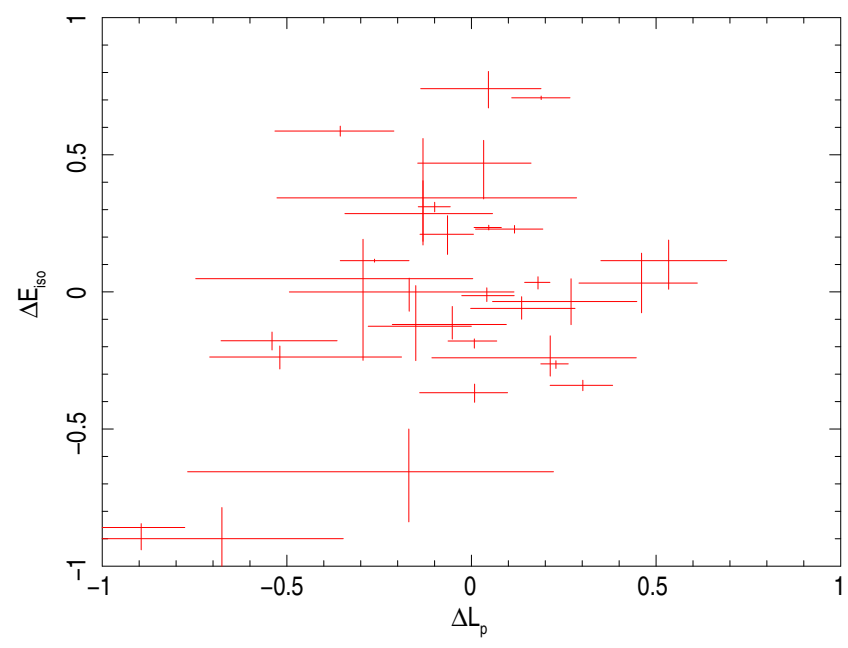

Figure 2. Residuals from calibrated Yonetoku relation and Amati relation from the observed $E_{p}$. Here, the residuals are the differences between the observed quantities, $L_{p}^{\text {obs }}$ and $E_{\text {iso }}^{\text {obs }}$, and the expected quantities, $L_{p}^{\exp }$ and $E_{\text {iso }}^{\text {exp }}$, from Yonetoku and Amati relations for the given observed $E_{p}$, respectively. There seems to be no correlation, which implies the two relations are independent distance indicators.

relations (the parameter $1-3$ and $2-3$ relations). This fact indicates that two distance indicators may be independent from each other. In the next section ( $\S-3)$, we investigate whether the cosmological parameters measured with two independent relations are consistent or not. After that, in $\S-4$, we explore a hidden parameter which is the cause of the intrinsic dispersion of Amati and Yonetoku relations.

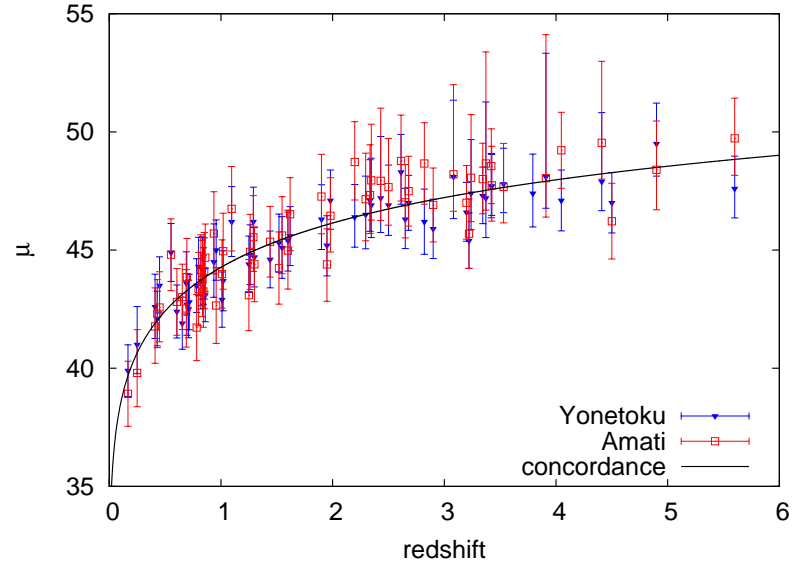

Figure 3. Extended Hubble diagram from Yonetoku relation (blue) and Amati relation (red). A systematic difference seems to exist in high redshift, although it doesn't seem in low redshift GRBs.

\section{CONSTRAINTS ON COSMOLOGICAL PARAMETERS}

We apply these Amati and Yonetoku relations to 29 GRBs with high redshifts, $1.9<z<5.6$, to determine the luminosity distance as a function of $z$. We include not only measurement errors of $E_{i s o}\left(L_{p}\right)$, and $E_{p}$ but also systematic errors we estimated in $\S-2$ and the 1-sigma uncertainties in Eq. (2) (Eq. (3)) to estimate luminosity distance (Schaefer 2007). For example, in case of Amati relation, we define $\mu_{0}\left(z_{i}\right)$ and $\sigma_{\mu_{0}, z_{i}}$ as below,

$$
\begin{aligned}
\mu_{0}\left(z_{i}\right)= & \frac{5}{2}\left(A+B \log E_{p}-\log \left(\frac{4 \pi S_{b o l}}{1+z}\right)\right) \\
\sigma_{\mu_{0}, z_{i}}^{2}= & \frac{25}{4}\left[\sigma_{A}^{2}+\left(\sigma_{B} \log E_{p}\right)^{2}+\sigma_{\text {sys }}^{2}\right. \\
& \left.+\left(0.434 B \sigma_{E p} / E_{p}\right)^{2}+\left(0.434 \sigma_{S_{b o l}} / S_{b o l}\right)^{2}\right] .
\end{aligned}
$$

Fig. 3 shows an extended Hubble diagram up to $z=5.6$ from Amati relation (red) and Yonetoku relation (blue). A systematic difference between red and green points seems to exist especially in high-redshift region.

Then we derive constraints on cosmological parameters. In the $\Lambda$-CDM model with $\Omega_{k}=\Omega_{m}+\Omega_{\Lambda}-1$, the luminosity distance is given by,

$$
\begin{aligned}
& d_{L}^{\text {th }}\left(z, \Omega_{m}, \Omega_{\Lambda}\right) \\
& \quad=(1+z) \begin{cases}\frac{c}{H_{0} \sqrt{\Omega_{k}}} \sin \left(\sqrt{\Omega_{k}} F(z)\right) & \text { if } \Omega_{k}>0 \\
\frac{c}{H_{0} \sqrt{-\Omega_{k}}} \sinh \left(\sqrt{-\Omega_{k}} F(z)\right) & \text { if } \left.\Omega_{k}<06\right) \\
\frac{c}{H_{0}} F(z) & \text { if } \Omega_{k}=0\end{cases}
\end{aligned}
$$

with

$F(z)=\int_{0}^{z} d z^{\prime}\left[\Omega_{m}\left(1+z^{\prime}\right)^{3}-\Omega_{k}\left(1+z^{\prime}\right)^{2}+\Omega_{\Lambda}\right]^{-1 / 2}$.

The likelihood contour is defined by,

$\Delta \chi^{2}=\sum_{i}\left\{\frac{\mu_{0}\left(z_{i}\right)-\mu^{\mathrm{th}}\left(z_{i}, \Omega_{m}, \Omega_{\Lambda},\right)}{\sigma_{\mu_{0, z_{i}}}}\right\}^{2}-\chi_{\text {best }}^{2}$,

where $\mu^{\text {th }}\left(z_{i}, \Omega_{m}, \Omega_{\Lambda}\right)=5 \log \left(d_{L}^{\text {th }} / \mathrm{Mpc}\right)+25$ and $\chi_{\text {best }}^{2}$ rep- 


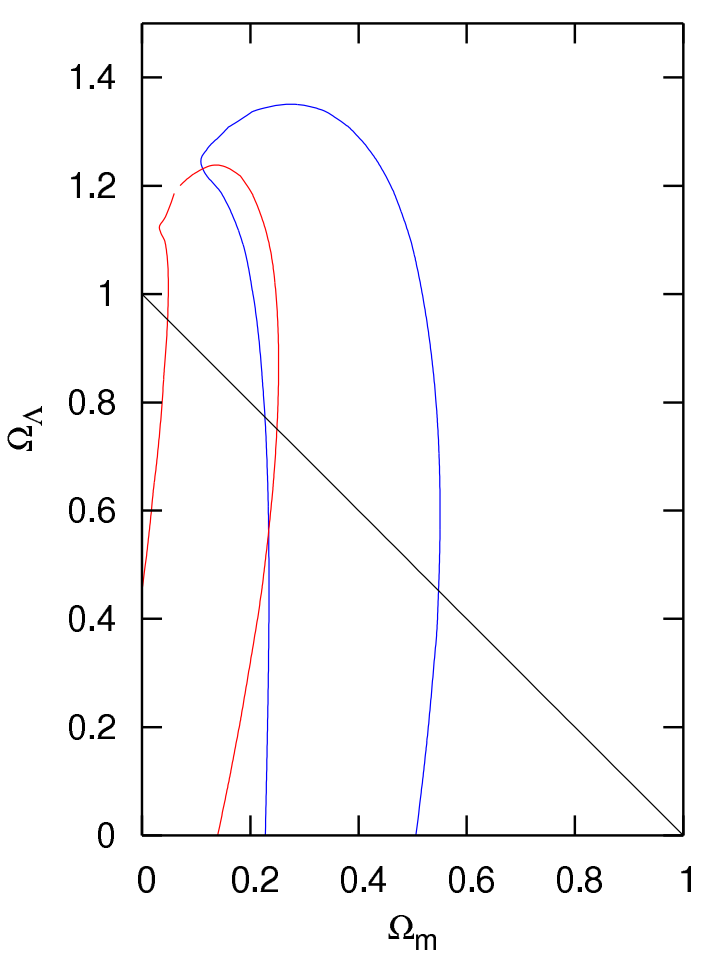

Figure 4. Constraints on $\left(\Omega_{m}, \Omega_{\Lambda}\right)$ plane from Amati relation (red) and Yonetoku relation (blue). The contours correspond to $68.3 \%$ confidence regions, and black solid line represents the flat universe. They are slightly different, although they are consistent in 2- $\sigma$ level. See also Table 1

\begin{tabular}{cccc}
\hline & $\Omega_{m}$ & $\Omega_{\Lambda}$ & $\chi_{\nu}^{2}$ \\
\hline Amati & $0.10_{--}^{+0.15}$ & $1.15_{--}^{0.09}$ & $11.46 / 27$ \\
Amati (flat) & $0.12_{-0.07}^{+0.13}$ & - & $11.69 / 28$ \\
\hline Yonetoku & $0.25_{-0.15}^{+0.31}$ & $1.24_{--}^{+0.11}$ & $9.36 / 28$ \\
Yonetoku (flat) & $0.34_{-0.14}^{+0.19}$ & - & $9.71 / 29$ \\
\hline$E_{p}-T_{L}-L_{p}$ & $0.17_{-0.08}^{+0.15}$ & $1.21_{-0.61}^{+0.07}$ & $16.59 / 27$ \\
$E_{p}-T_{L}-L_{p}$ (flat) & $0.24_{-0.09}^{+0.11}$ & - & $17.50 / 28$ \\
\hline
\end{tabular}

Table 1. Constraints on $\left(\Omega_{m}, \Omega_{\Lambda}\right)$ in non-flat and flat universe with 1- $\sigma$ errors from Amati, Yonetoku and $E_{p}-T_{L}-L_{p}$ relations and their reduced chi squares. Constraints from Amati and Yonetoku relations are inconsistent in $1-\sigma$ level.

resents the chi-square value for the best-fit parameter set of $\Omega_{m}$ and $\Omega_{\Lambda}$.

In Fig. 4, we show the likelihood contour from Amati (red) and Yonetoku relations (blue), and the best-fit values with 1- $\sigma$ errors are shown in Table 1 Interestingly, they are slightly different, although they are consistent in $2-\sigma$ level.

\section{NEW RELATION}

In this section, we seek for a hidden parameter which reduces the systematic error in the relation. Note that the discussion in this section does not concern with independence of the two relations. If we could find this parameter, we can make strong constraint on cosmological param- eters, because the systematic errors in the Amati and Yonetoku relations are larger than their measurement errors. Ghirlanda et al. 2006; Firmani et al. 2006) There are some studies using jet break time in afterglow as such a parameter (Ghirlanda et al. 2004; Liang \& Zhang 2005), but the number of GRBs which have observed jet is small. Here we restrict our discussion in a prompt emission property. In the past studies, prompt emission was characterized by a time scale, the duration of most intense parts of the GRB $\left(T_{0.45}\right)$ (Firmani et al. 2006; Rossi et al. 2008; Collazzi \& Schaefer 2008). Here we adopt a time scale called luminosity time $T_{L}$ introduced by Willingale et al. (2007) as,

$T_{L}=\frac{E_{\text {iso }}}{L_{p}}=\frac{S_{\mathrm{bol}}}{(1+z) F_{p}}$.

The luminosity time does not depend on detector's energy band because it is defined by bolometric flux and fluence.

We assume the correlation among $E_{p}, T_{L}$ and $L_{p}$ to be of the form, $\log L_{p} \equiv A+B \log E_{p}+C \log T_{L}$. Then we obtain,

$\frac{L_{p}}{10^{52} \mathrm{erg} \mathrm{s}^{-1}}=10^{-3.87 \pm 0.19}\left(\frac{E_{p}}{1 \mathrm{keV}}\right)^{1.82 \pm 0.08}\left(\frac{T_{L}}{1 \mathrm{~s}}\right)^{-0.34 \pm 0.09}$

from low redshift $30 \mathrm{GRBs}$ in $0.16<z<1.7$. In Fig. 5 we show $E_{p}-T_{L}-L_{p}$ relation. The Pearson correlation coefficient is 0.971 , the reduced chi-square is $\chi^{2}=26.9 / 27$ with the systematic error $\sigma^{\text {sys }}=0.15$. We include not only errors in $L_{p}$ but also errors in $E_{p}$, and $T_{L}$ so the chi-square function is defined as $\chi^{2}(A, B, C)=$ $\Sigma\left(\log L_{p}^{o b s}-A-B \log E_{p}-C \log T_{L}\right)^{2} /\left(\sigma_{\text {meas }}^{2}+\sigma_{\text {sys }}^{2}\right)$ where the weighting factor $\sigma_{\text {meas }}^{2}=(1+2 C) \sigma_{\log L_{p}}^{2}+$ $\left(B \sigma_{\log E_{p}}\right)^{2}+\left(C \sigma_{\log T_{L}}\right)^{2}$, and $\sigma_{\log T L}$ is estimated by using the error propagation equation without a crossterm between $L_{p}$ and $E_{\text {iso. }}$. The factor $2 \mathrm{C}$ in the front of $\sigma_{\log L_{p}}^{2}$ comes from the fact that the definition of $T_{L}$ includes $L_{p}$. Note that the contribution of the additional error term $\sigma_{T_{L}}$ to chi-square value is little because of its small slope. Thus, we conclude that the additional term really improves relations. The systematic error is substantially reduced compared to those of Amati and Yonetoku relations, and now comparable to the measurement error. Thus this relation could be regarded as "Fundamental plane" (Diorgovski \& Davis 1987) of GRB prompt emission.

Here we excluded one outlier, GRB070521, from the fitting of Eq. (10). Actually, the host galaxy of GRB070521 is detected inside an error circle of XRT by Hattori, T., Aoki, K., \& Kawai, N. (2007) using Subaru Telescope after 40 minutes from the trigger, but they couldn't detect bright afterglow. Thus, the real redshift may be larger, which is why we exclude GRB070521 from our analysis. Note that the probability of the miss identification of the host galaxy from only XRT observation is about $7 \%$ Cobb \& Bailvn 2008). Futhermore there might be another population of GRBs so that the secure classification of GRBs is one of the current issue of GRB's study. For more detailed discussions about $E_{p}-T_{L}-L_{p}$ relation, see Tsutsui et al. (in prep).

We show a possible derivation of the new relation (Eq.110) under the photospheric model of the prompt emission of GRBs ( Ioka et al. (2007) and references therein). The luminosity is given by

$L \propto r^{2} \Gamma^{2} T^{4}$ 


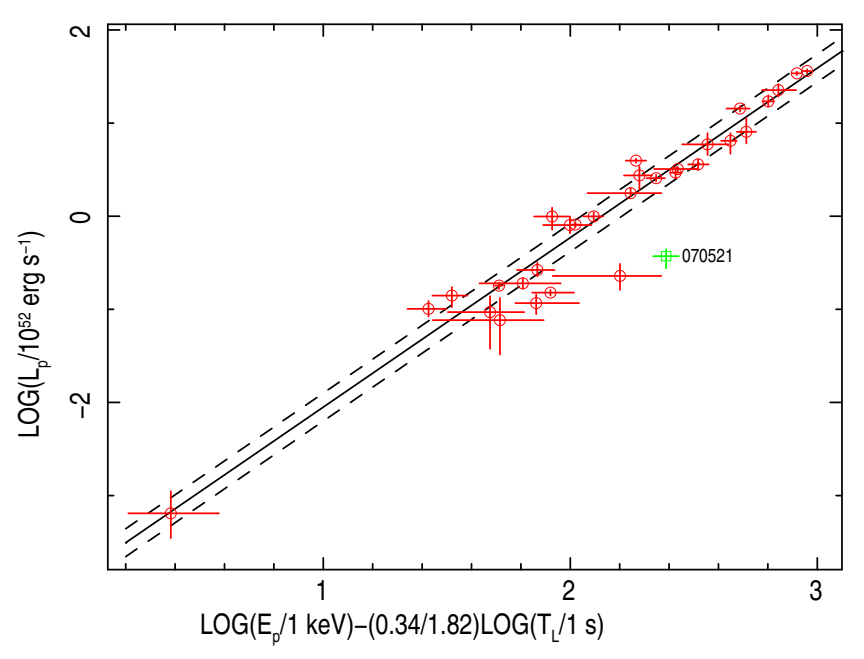

Figure 5. The $E_{p} / T_{L}^{0.187}$ and $L_{p}$ in 31 GRBs with $z<1.62$. The correlation is improved than Amati and Yonetoku relations. The solid line shows the best-fit curve without one outlier (green square:GRB070521), and dashed lines represent the 1-sigma region. The Pearson correlation coefficient is 0.971 and reduced chisquare is $\chi^{2}=26.9 / 27$ with the systematic error $\sigma_{\text {sys }}=0.15$ which is comparable to the measurement error.

where $r, \Gamma$ and $T^{\prime}$ are the photospheric radius, the gamma factor and co-moving temperature of the photosphere, respectively. Since Ioka et al. (2007) assume that the energy is supplied by the relativistic collision of the rapid shell of mass $m_{r}$ and Lorenz factor $\gamma_{r}$ with the slow shell of $m_{s}$ and $\gamma_{s}$. Then under the perfectly inelastic collision model, $\Gamma$ is given by $\Gamma^{2}=\left(m_{r} \gamma_{r}+m_{s} \gamma_{s}\right) /\left(m_{r} / \gamma_{r}+m_{s} / \gamma_{s}\right)$. Since $\gamma_{r} \gg \gamma_{s}$ and $m_{r} \gamma_{r}+m_{s} \gamma_{s} \propto E_{i s o}$, we can reduce $\Gamma^{2} \propto$ $E_{\text {iso }} \gamma_{s} / m_{s}$. If we regard $E_{p} \sim \Gamma T^{\prime}$, we can rewrite Eq. (11) as $L \propto r^{2} E_{p}^{4} / \Gamma^{2} \propto r^{2} m_{s} E_{p}^{4} / E_{i s o} \gamma_{s}$. Now let us assume $r$, $m_{s}$ and $\gamma_{s}^{2} T_{L}$ are constants. Then we have $L \propto E_{p}^{2} T_{L}^{-0.25}$. The above relation is essentially the same as Eq.(10) if we consider 2- $\sigma$ error of the power index in Eq. (10).

Possible reasons for the above three assumptions are as follows. In Ioka et al. (2007) model $r$ is similar to the radius of the progenitor star so that it could be constant. $T_{L}$ can be regarded as the effective duration of the burst. Then $c \gamma_{s}^{2} T_{L}$ is the radius that the last rapid shell catches the slow shell and we expect that this is also the order of the radius of the progenitor star, which is constant. We have no reason why $m_{s}$ is constant. However if $m_{s}$ obeys the log normal distribution like the other observables in GRBs we may regard it essentially constant. If these assumptions are reasonable, the new relation (Eq. (10) could be derived in the photospheric model of the prompt emission of GRBs like Ioka et al. (2007).

Finally we use this new relation to put constraints on cosmological parameters. Here again we include not only measurement errors of $L_{p}, E_{p}, T_{L}$ but also systematic errors and the 1-sigma uncertainties in Eq.110 to estimate luminosity distance (Schaefer 2007).

The concordance cosmology is still consistent in 1$\sigma$ level. The constraints on cosmological parameters are $\left(\Omega_{m}, \Omega_{\Lambda}\right)=\left(0.17_{-0.08}^{+0.15}, 1.21_{-0.61}^{+0.07}\right)$ and $\chi_{\nu}^{2}=16.59 / 27$ for non-flat universe, $\Omega_{m}=0.24_{-0.09}^{+0.11}$ and $\chi_{\nu}^{2}=17.50 / 28$ for flat universe. (See Table 1)

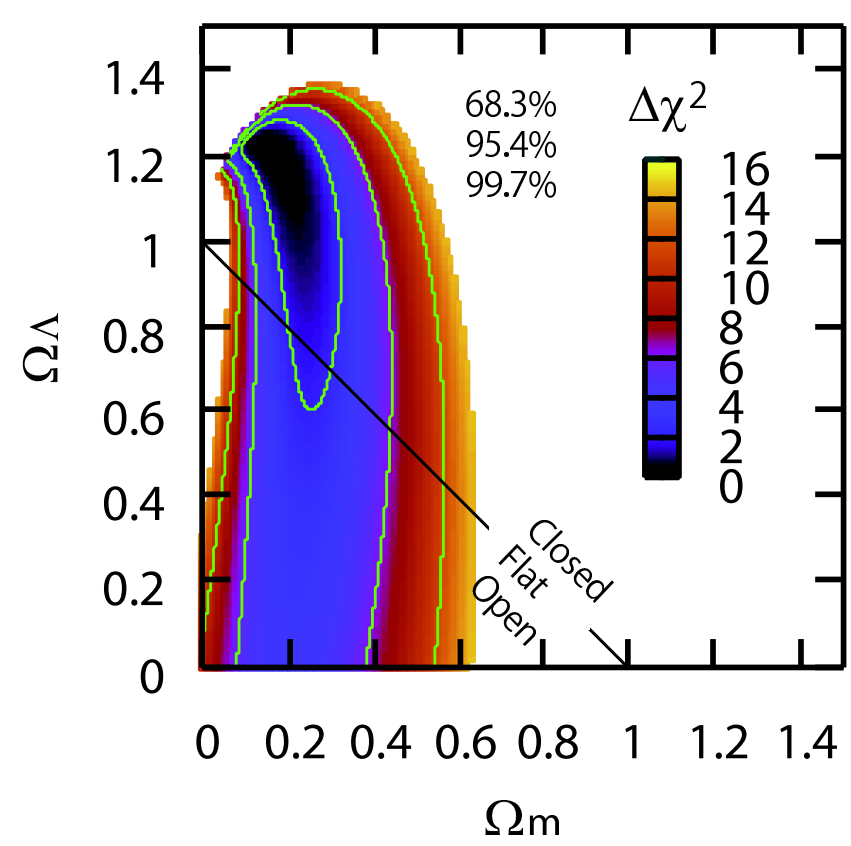

Figure 6. Constraint on $\left(\Omega_{m}, \Omega_{\Lambda}\right)$ plane from $E_{p}-T_{L}-L_{p}$ relation. See also Table 1

\section{DISCUSSION}

Recently some authors extended Hubble diagram up to $z \sim 6$ using various luminosity indicators (Amati et al. 2008; Liang et al. 2008; Schaefer 2007; Firmani et al. 2006; Ghirlanda et al. 2006). Ghirlanda et al. (2006), Firmani et al. (2006), and Schaefer (2007) are pioneering works for GRB cosmology, but they are caught in circularity problem because there are few GRBs at low redshift.

Schaefer (2007) obtained cosmological constraints from lag $\left(\tau_{\text {lag }}\right)$-luminosity relation, variability $(V)$-luminosity relation, $E_{p}$-jet collimated energy $\left(E_{\gamma}\right)$ relation (so called Ghirlanda relation), minimum rise time $\left(\tau_{\mathrm{RT}}\right)$-luminosity relation, and Yonetoku relation. Liang et al. (2008) calibrated these relations by luminosity distances from SNIa observations.

However, Tsutsui et al. (2008) showed redshift dependence of $\tau_{\text {lag }}-L_{p}$ relation analyzing 565 BASTE GRB samples with pseudo-redshifts estimated by Yonetoku relation. This suggests that the relation cannot be used as a distance indicator. The dependence might come from the fact that $\tau_{\text {lags }}$ were evaluated from several fixed energy bands depending on the detectors and they are different energy in GRB rest frame, so it suffer from K-correction problem. This argument applies to $V \mathrm{~s}$ and $\tau_{\mathrm{RT}} \mathrm{S}$.

Although $E_{p}-E_{\gamma}$ relation and Liang \& Zhang relation have much smaller systematic errors than both Yonetoku and Amati relations, there are many GRBs without a jet break or with multiple jet breaks (missing or multiple jet break problem) so that it is not certain whether the jet break can be used to characterize GRB emission. In contrast, our new relation does not suffer from these problems, because it is totally defined by the prompt emission property and it has as a small systematic error as $E_{p}-E_{\gamma}$ and Liang \& Zhang relations. Thus we could expect that the new relation would put GRB cosmology to the next promising 
stage, as Phillips relation (Phillips 1993) and Fundamental plane (Diorgovski \& Davis 1987) did for SNeIa and elliptical galaxies. However, we must emphasize that we need detailed studies of the new relation with much larger number of GRBs and examination of systematic errors in order for GRB to be regarded as a reliable tool for cosmology like SNIa, cosmic microwave background, baryon acoustic oscillation and gravitational lens. Now ongoing Missions like Swift , Fermi and Suzaku, and the collaboration of many observer on ground will promise the progression of GRB cosmology.

\section{ACKNOWLEDGMENTS}

This work is supported in part by the Grant-in-Aid from the Ministry of Education, Culture, Sports, Science and Technology (MEXT) of Japan, No.19540283, No.19047004(TN), and No.20674002 (DY) and by the Grant-in-Aid for the global COE program The Next Generation of Physics, Spun from Universality and Emergence from MEXT of Japan. KT is supported in part by a Grant-in-Aid from the Japan Society for the Promotion of Science (JSPS) Fellows and the global COE programs, "Quest for Fundamental Principles in the Universe: from Particles to the Solar System and the Cosmos" at Nagoya University.

\section{REFERENCES}

Amati, L., et al. 2002, AAP, 390, 81

Amati, L., Guidorzi, C., Frontera, F., Della Valle, M., Finelli, F., Landi, R., \& Montanari, E. 2008, ArXiv eprints, 805, arXiv:0805.0377

Band, D., et al. 1993, ApJ, 413, 281

Basilakos, S., \& Perivolaropoulos, L. 2008, MNRAS, 391, 411

Bevington, P. R., \& Robinson, D. K. 2003, Data reduction and error analysis for the physical sciences, 3rd ed., by Philip R. Bevington, and Keith D. Robinson. Boston, MA: McGraw-Hill, ISBN 0-07-247227-8, 2003.,

Butler, N. R., Kocevski, D., Bloom, J. S., \& Curtis, J. L. 2007, ApJ, 671, 656

Cobb, B. E., \& Bailyn, C. D. 2008, ApJ, 677, 1157

Collazzi, A. C., \& Schaefer, B. E. 2008, ApJ, 688, 456

Davis, T. M., et al. 2007, ApJ, 666, 716

Djorgovski, S., \& Davis, M. 1987, ApJ, 313, 59

Firmani, C., Avila-Reese, V., Ghisellini, G., \& Ghirlanda, G. 2006, MNRAS, 372, L28

Firmani, C., Ghisellini, G., Avila-Reese, V., \& Ghirlanda, G. 2006, MNRAS, 370, 185

Ghirlanda, G., Ghisellini, G., \& Lazzati, D. 2004, ApJ, 616, 331

Ghirlanda, G., Ghisellini, G., \& Firmani, C. 2006, New Journal of Physics, 8, 123

Hattori, T., Aoki, K., \& Kawai, N. 2007, GCN 6444

Ioka, K., et al. 2007, ApJ, 670, L77-L80

Kodama, Y., Yonetoku, D., Murakami, T., Tanabe, S., Tsutsui, R., \& Nakamura, T. 2008, MNRAS, 391, L1

Li, L.-X. 2007, MNRAS, 379, L55

Liang, E., \& Zhang, B. 2005, ApJ, 633, 611
Liang, N., Xiao, W. K., Liu, Y., \& Zhang, S. N. 2008, ArXiv e-prints, 802, arXiv:0802.4262

Phillips, M. M. 1993, ApJL, 413, L105

Riess, A. G., et al. 2007, ApJ, 659, 98

Rossi, F., et al. 2008, MNRAS, 388, 1284

Schaefer, B. E. 2007, ApJ, 660, 16

Tsutsui, R., Nakamura, T., Yonetoku, D., Murakami, T.,

Tanabe, S., \& Kodama, Y. 2008, MNRAS, 386, L33

Tsutsui, R., Nakamura, T., Yonetoku, D., Murakami, T., Tanabe, S., Kodama, Y., \& Takahashi, K. 2009, MNRAS, 394, L31

Tsutsui, R., Nakamura, T., Yonetoku, D., Murakami, T., Tanabe, S., Kodama, Y., \& Takahashi, K. 2008, in preperation

Willingale, R., O'Brien, P. T., Goad, M. R., Osborne, J. P., Page, K. L., \& Tanvir, N. R. 2007, ArXiv e-prints, 710, arXiv:0710.3727

Wood-Vasey, W. M., et al. 2007, ApJ, 666, 694

Yonetoku, D., Murakami, T., Nakamura, T., Yamazaki, R., Inoue, A. K., \& Ioka, K. 2004, ApJ, 609, 935 